ARTICLE

Received 19 Jul 2013 | Accepted 3 Dec 2013 | Published 10 Jan $2014 \quad$ DOl: 10.1038/ncomms4053

\title{
A fluorinated dendrimer achieves excellent gene transfection efficacy at extremely low nitrogen to phosphorus ratios
}

Mingming Wang ${ }^{1, \star}$, Hongmei Liu ${ }^{1, \star}$, Lei Li $i^{1} \&$ Yiyun Cheng ${ }^{1}$

Polymers have shown great promise in the design of high efficient and low cytotoxic gene vectors. Here we synthesize fluorinated dendrimers for use as gene vectors. Fluorinated dendrimers achieve excellent gene transfection efficacy in several cell lines (higher than $90 \%$ in HEK293 and HeLa cells) at extremely low N/P ratios. These polymers show superior efficacy and biocompatibility compared with several commercial transfection reagents such as Lipofectamine 2000 and SuperFect. Fluorination enhances the cellular uptake of the dendrimer/DNA polyplexes and facilitates their endosomal escape. In addition, the fluorinated dendrimer shows excellent serum resistance and exhibits high gene transfection efficacy even in medium containing 50\% FBS. The results suggest that fluorinated dendrimers are a new class of highly efficient gene vectors and fluorination is a promising strategy to design gene vectors without involving sophisticated syntheses.

\footnotetext{
${ }^{1}$ Shanghai Key Laboratory of Regulatory Biology, School of Life Sciences, East China Normal University, Shanghai 200241, China. ${ }^{\star}$ These authors contributed equally on this work. Correspondence and requests for materials should be addressed to Y.C. (email: yycheng@mail.ustc.edu.cn).
} 
C ationic polymers are widely used as promising gene vectors to introduce exogenous nucleic acid into cells ${ }^{1-7}$. These materials are easy-to-manufacture and have superior safety compared with viral gene vectors; however, their commercial and clinical applications are limited by relatively low transfection efficacy and poor cell viability after transfection $^{8-11}$. Functionalization of polymers with amino acids $^{12}$, lipids ${ }^{13}$, cyclodextrins ${ }^{14}$, peptides ${ }^{15}$, other polymers ${ }^{16}$ and inorganic nanoparticles ${ }^{17}$ can improve their transfection efficacy and biocompatibility to some extent, but the resulting materials are still not efficient enough to justify clinical uses.

Fluorine is absent in biological systems, but is widely used to tailor the properties or behaviours of drugs, biomacromolecules and cells ${ }^{18-22}$. Fluorination increases the therapeutic efficacy or pharmacokinetic behaviour of numerous drugs ${ }^{18,19}$, improves the stability of proteins without altering their structures and functions ${ }^{21}$, and allows the detection or imaging of the labelled compounds using magnetic resonance imaging or positron emission tomography imaging 23,24 . Fluorinated compounds are both hydrophobic and lipophobic, and have a high phase-separation tendency in both polar and non-polar environments ${ }^{25,26}$. Therefore, fluorination can improve the affinity of polymers to cell membrane ${ }^{27}$ and across the lipid bilayer of the cell membrane, as well as the endosome/lysosome membrane ${ }^{28}$, facilitating endosomal escape of the polymers. In addition, fluorinated polymers have low surface energy and prefer to associate with each other at low concentrations ${ }^{29-31}$, allowing the formation of polyplexes with nucleic acid at low nitrogen to phosphorus (N/P) ratios. Gene transfection at a low $\mathrm{N} / \mathrm{P}$ ratio is essential to minimize the toxicity on the transfected cells. Dendrimers with versatile properties are frequently used in the field of biomaterials and nanomedicine ${ }^{32-37}$. These polymers are monodisperse and can be easily modified with fluorous compounds ${ }^{38,39}$.

Here we propose a fluorination strategy to dramatically improve the efficacy of cationic polymers while reducing their cytotoxicity in gene transfection. We synthesize fluorinated dendrimers by a facile route and use them as a new class of polymeric gene vectors. The fluorinated dendrimers fulfill the two main requirements in gene delivery-dramatic transfection efficacy and low cytotoxicity. Fluorination also works well on other cationic polymers such as poly(ethylenimine) in gene delivery and represents a promising tool in the design of high efficient and low cytotoxic gene materials.

\section{Results}

Characterization of fluorinated dendrimers. Generation 5 (G5) poly(amidoamine) (PAMAM) dendrimers were reacted with perfluoro acid anhydrides at room temperature to yield fluorinated dendrimers (Fig. 1a). The number of perfluoro acid modified on each G5 dendrimer is evaluated by a well-established

a

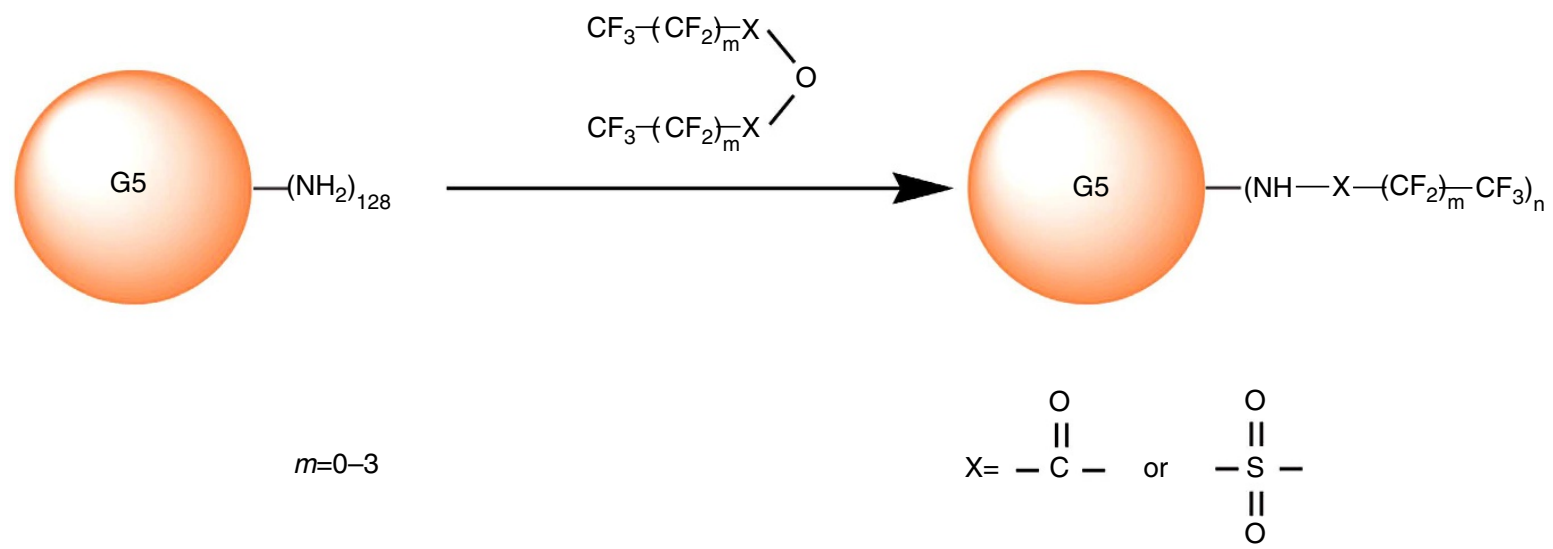

b
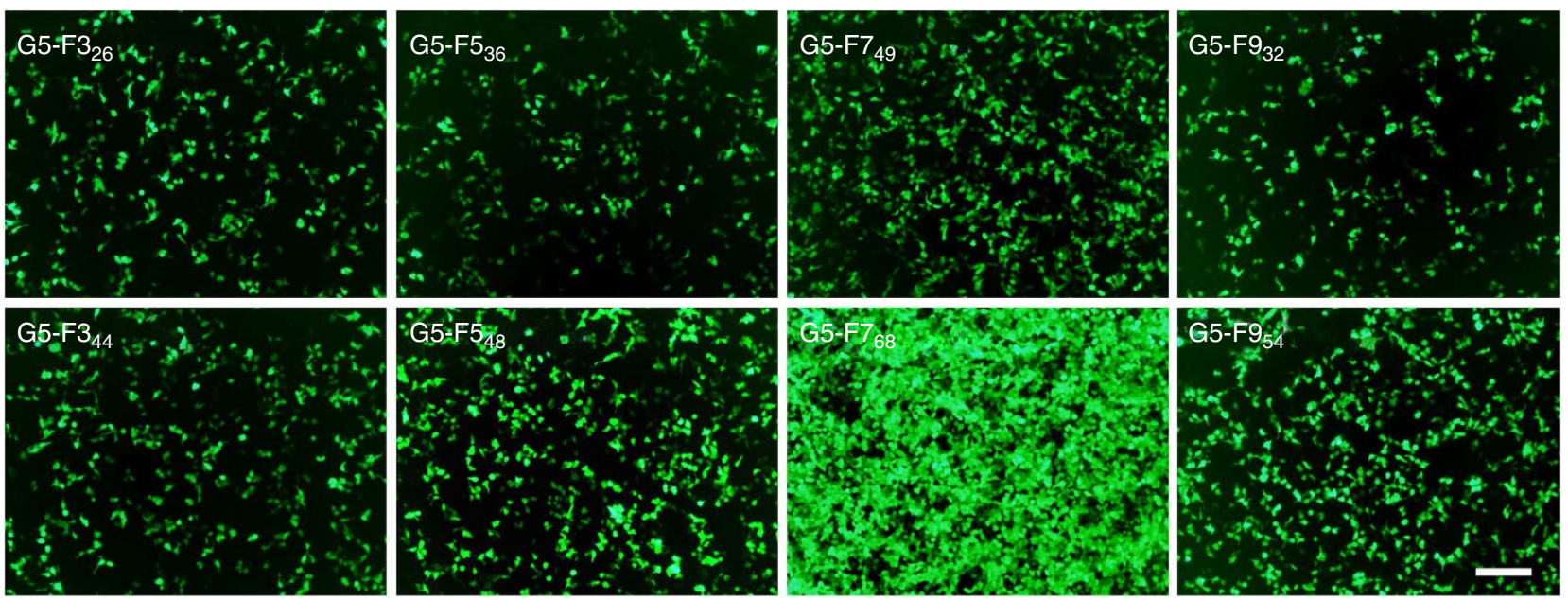

Figure 1 | Synthesis and gene transfection efficacy of fluorinated dendrimers. (a) Synthetic route of the fluorinated dendrimers. (b) EGFP gene transfection efficacies of the synthesized fluorinated dendrimers in HEK293 cells at their optimized N/P ratios listed in Supplementary Fig. 1. Scale bar, $200 \mu \mathrm{m}$ 
ninhydrin assay (Supplementary Table 1). The resulting materials are termed $\mathrm{G} 5-\mathrm{F}_{26}, \mathrm{G} 5-\mathrm{F}_{44}, \mathrm{G} 5-\mathrm{F} 5_{36}, \mathrm{G} 5-\mathrm{F}_{48}, \mathrm{G} 5-\mathrm{F} 7_{49}$, $\mathrm{G} 5-\mathrm{F}_{68}, \mathrm{G} 5-\mathrm{F}_{32}$ and $\mathrm{G} 5-\mathrm{F9}_{54}$, respectively. Gene transfection efficacy of these fluorinated dendrimers at their optimized N/P ratios were tested in HEK293 cells using an enhanced green fluorescent protein (EGFP) plasmid. As shown in Fig. $1 \mathrm{~b}$ and Supplementary Fig. 1, heptafluorobutyric acid-modified dendrimers (G5-F7 68 and G5-F7 ${ }_{49}$ ) show much higher transfection efficacy in HEK293 cells than other fluorinated dendrimers. Therefore, G5-F7 68 and $\mathrm{G} 5-\mathrm{F}_{49}$ were investigated in detail in further gene transfection studies.

Fluorination improves transfection efficacy of dendrimers. As expected, both G5-F7 68 and G5-F7 49 effectively form polyplexes with DNA (Supplementary Fig. 2) and condense the plasmid DNA into $100-200 \mathrm{~nm}$ nanoparticles even at an extremely low N/P ratio of 0.5 (Supplementary Figs 3 and 4). Vierling et al. ${ }^{40}$ increased the transfection efficacy of cationic lipids through fluorination. However, the fluorinated cationic lipids must be typically co-formulated with non-fluorinated lipids and only show moderate increases in transfection efficacy ${ }^{40,41}$. Here, fluorination significantly improves the EGFP transfection efficacy of a G5 dendrimer (from 21.8 to $69.9 \%$ for G5-F7 49 and $97.1 \%$ for G5-F7 68 ) in HEK293 cells (Fig. 2a,b). High efficacy of G5-F7 68 achieves at an extremely low N/P ratio of 1.5, and its efficacy is higher than Lipofectamine 2000 (70.8\%), a commercial transfection reagent. Similar results were obtained using a luciferase reporter gene (Fig. 2c). The high transfection efficacy of G5-F7 68 is further confirmed in HeLa cells (Fig. 3 and Supplementary Fig. 5), COS-7 cells (Supplementary Fig. 6), CHO cells (Supplementary Fig. 7) and NIH3T3 cells (Supplementary Fig. 8). G5-F7 68 shows higher efficacies than Lipofectamine 2000 in most of the cells, except in CHO cells (Fig. 4). G5-F7 68 is also more efficient than SuperFect, branched poly(ethylenimine) with a molecular weight of $25 \mathrm{kDa}$ (bPEI25K), lysine- and argininemodified G5 dendrimers (G5-Lys 130 and G5-Arg ${ }_{108}$ ), which were shown to be effective in introducing DNA into cells such as HeLa cells (Fig. $4 \mathrm{~b})^{42}$. Even at an extremely low N/P ratio of 0.5 , relatively high EGFP gene expressions $(\sim 50 \%)$ in $\mathrm{HeLa}$ and HEK293 cells were observed for the G5-F7 ${ }_{68} / \mathrm{DNA}$ polyplexes (Supplementary Fig. 9 and Supplementary Note 1).

a
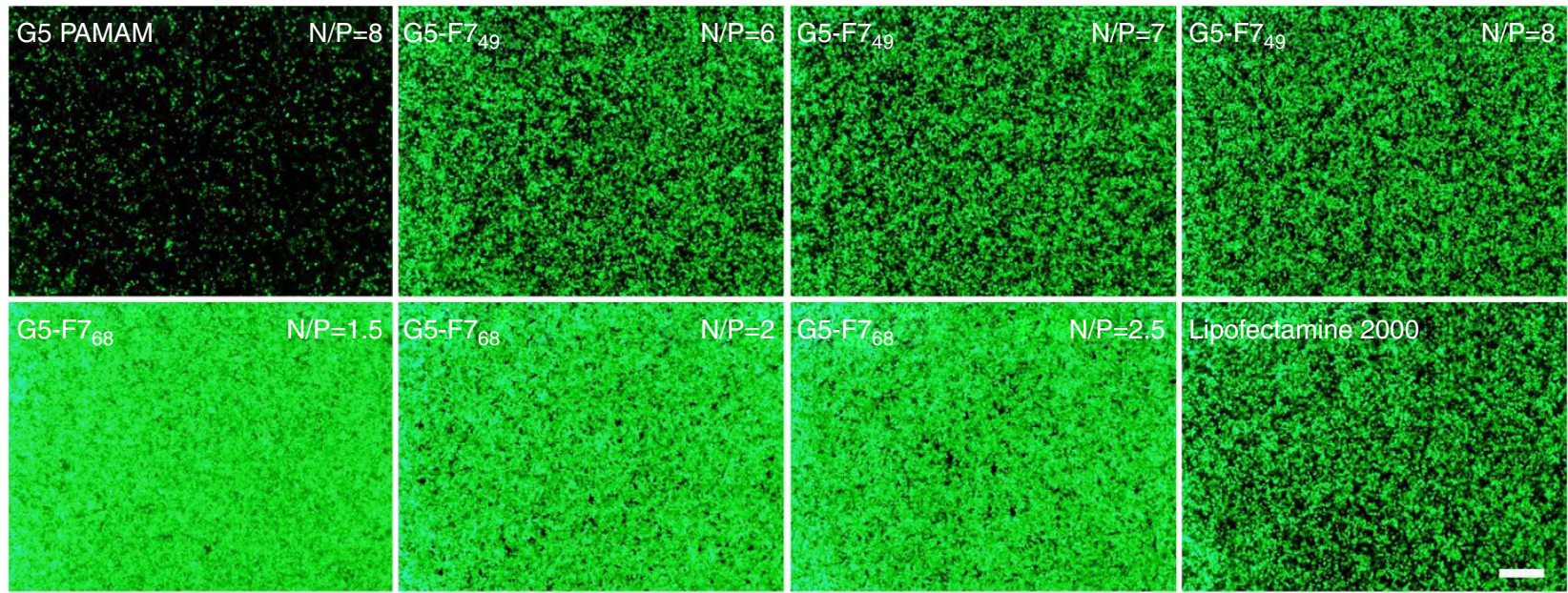

b

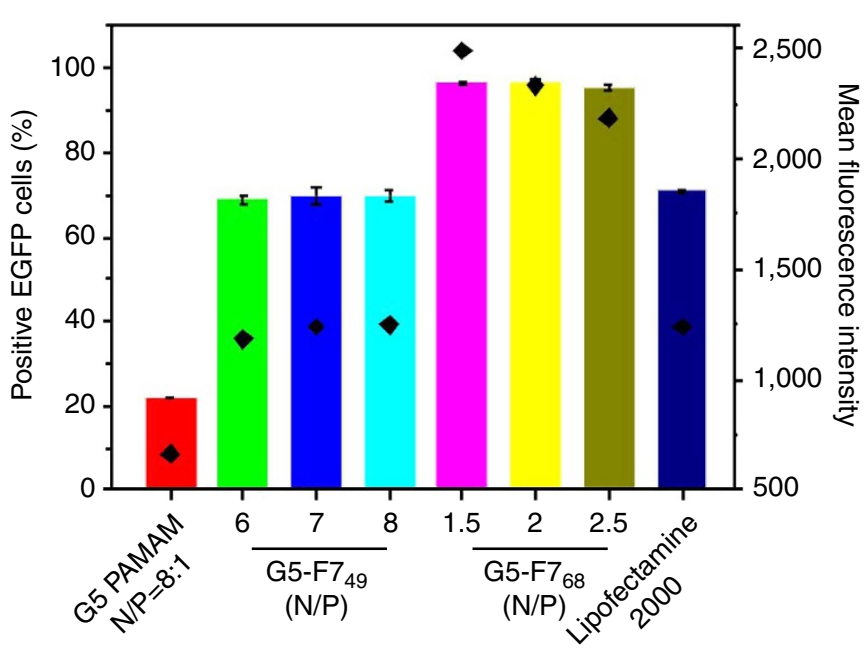

c

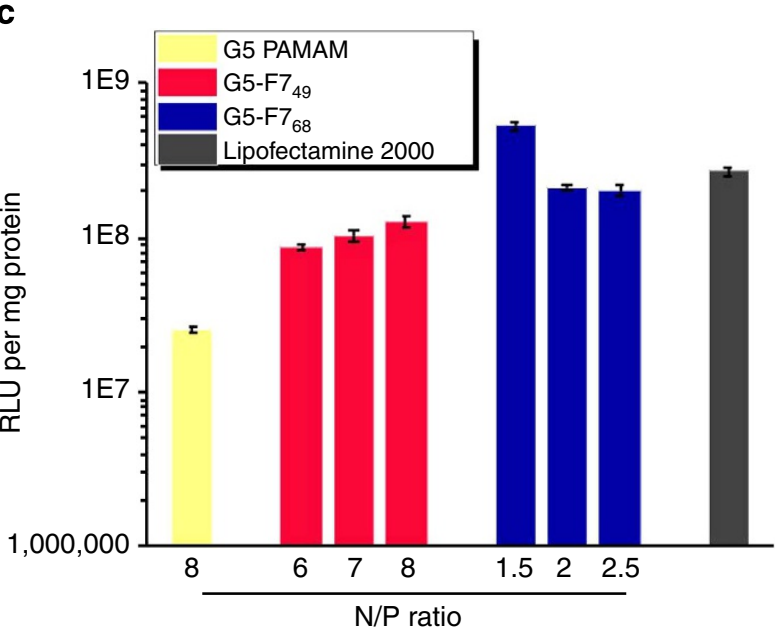

Figure 2 | Transfection efficacies of fluorinated dendrimers in HEK293 cells. Comparison of fluorinated dendrimers (G5-F7 49 and G5-F7 68 ) with G5 and Lipofectamine 2000 on the in vitro EGFP (a,b) and luciferase (c) gene transfection efficacy in HEK293 cells after 24 h. Positive EGFP cells (\%) were determined by flow cytometry (b) and the luciferase gene expression level (c) is expressed as relative luciferase light units per milligram of protein (RLU per $\mathrm{mg}$ protein). Error bars in $\mathbf{b}$ and $\mathbf{c}$ represent the s.e. $(n=3)$. Diamonds in $\mathbf{b}$ represent mean fluorescence intensity. Scale bar, $400 \mu \mathrm{m}$. 
a
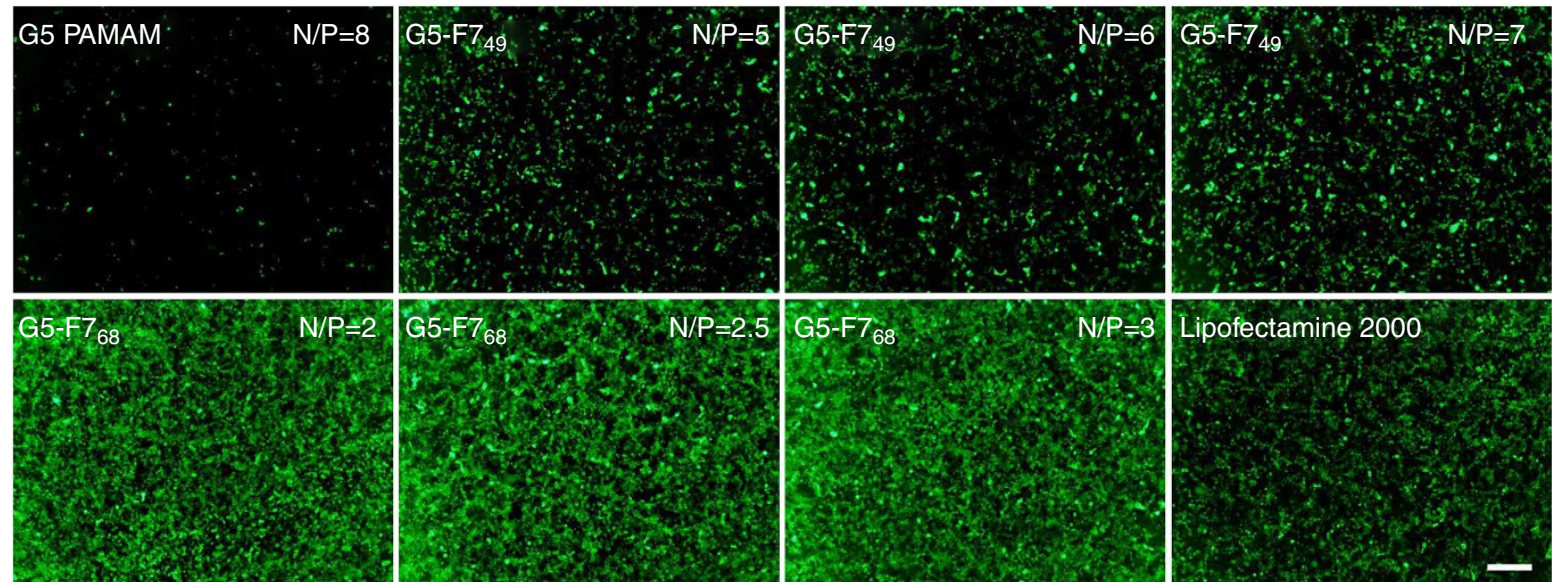

b

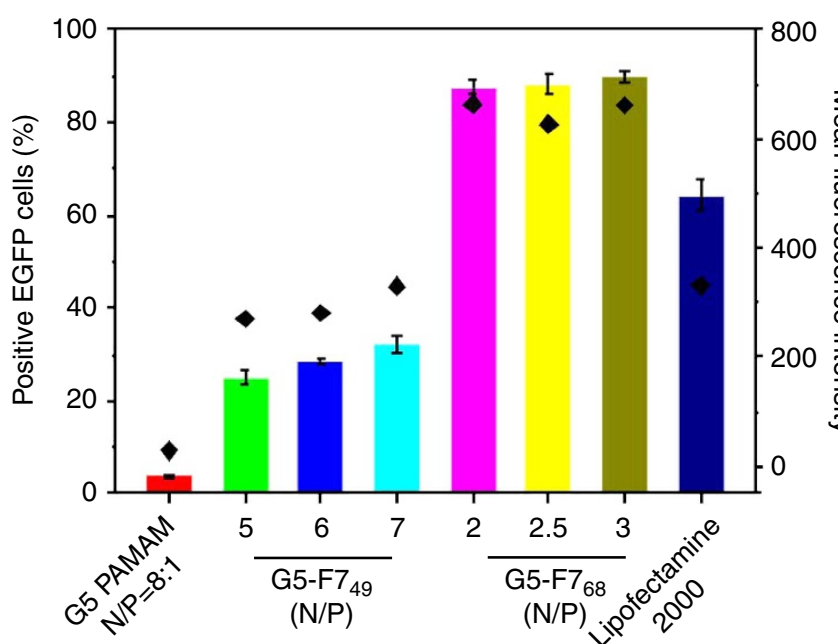

c

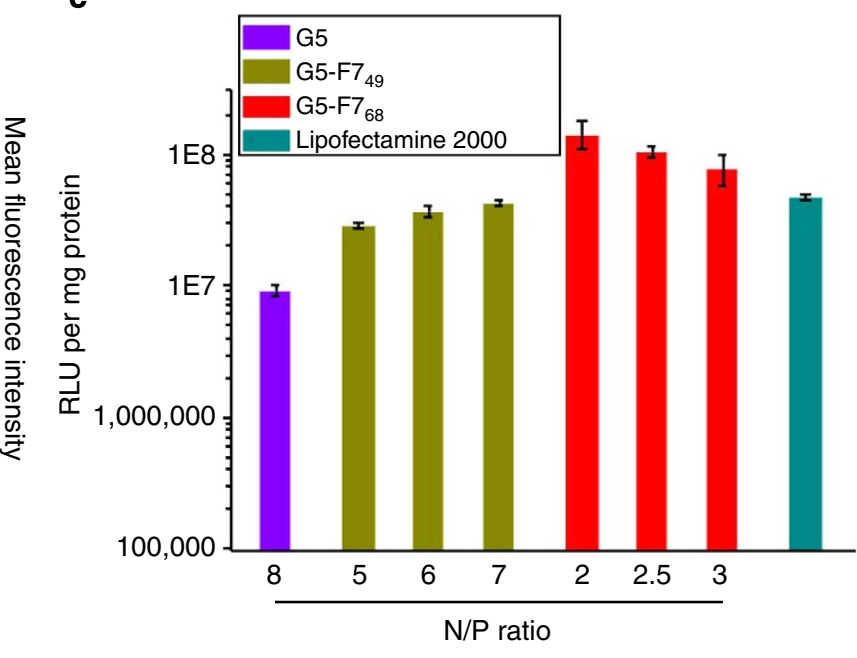

Figure 3 | Transfection efficacies of fluorinated dendrimers in HeLa cells. Comparison of fluorinated dendrimers with G5 and Lipofectamine 2000 on the in vitro EGFP $(\mathbf{a}, \mathbf{b})$ and luciferase (c) gene transfection efficacy in HeLa cells after $48 \mathrm{~h}$. Error bars in $\mathbf{b}$ and $\mathbf{c}$ represent the s.e. ( $n=3$ ). Diamonds in b represent mean fluorescence intensity. Scale bar, $400 \mu \mathrm{m}$.

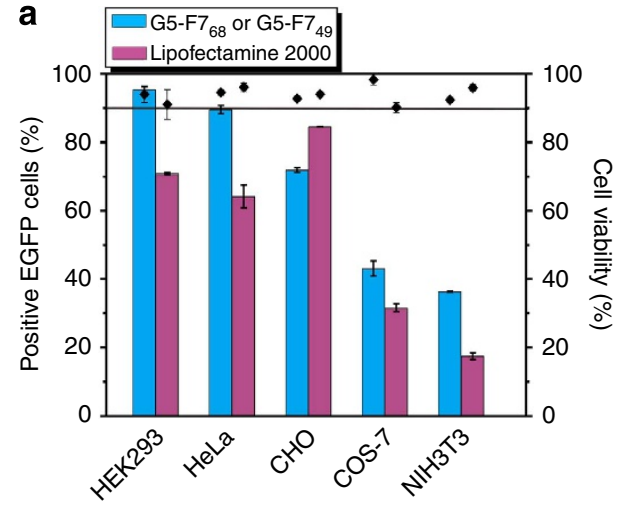

b

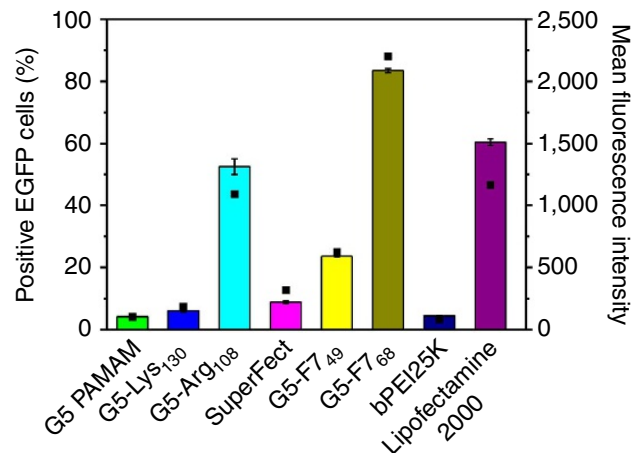

Figure 4 | Comparison of fluorinated dendrimers with other transfection reagents on gene transfection efficacy. EGFP expression and cell viability in five cell lines transfected by fluorinated dendrimers and Lipofectamine 2000 (a). Diamonds in a represent cell viability (\%). Comparison of

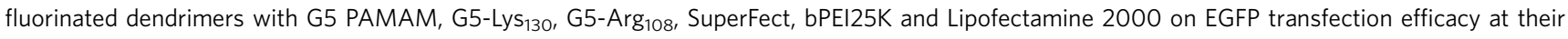
optimized condition in HeLa cells after $48 \mathrm{~h}(\mathbf{b})$. Squares in $\mathbf{b}$ represent mean fluorescence intensity. 

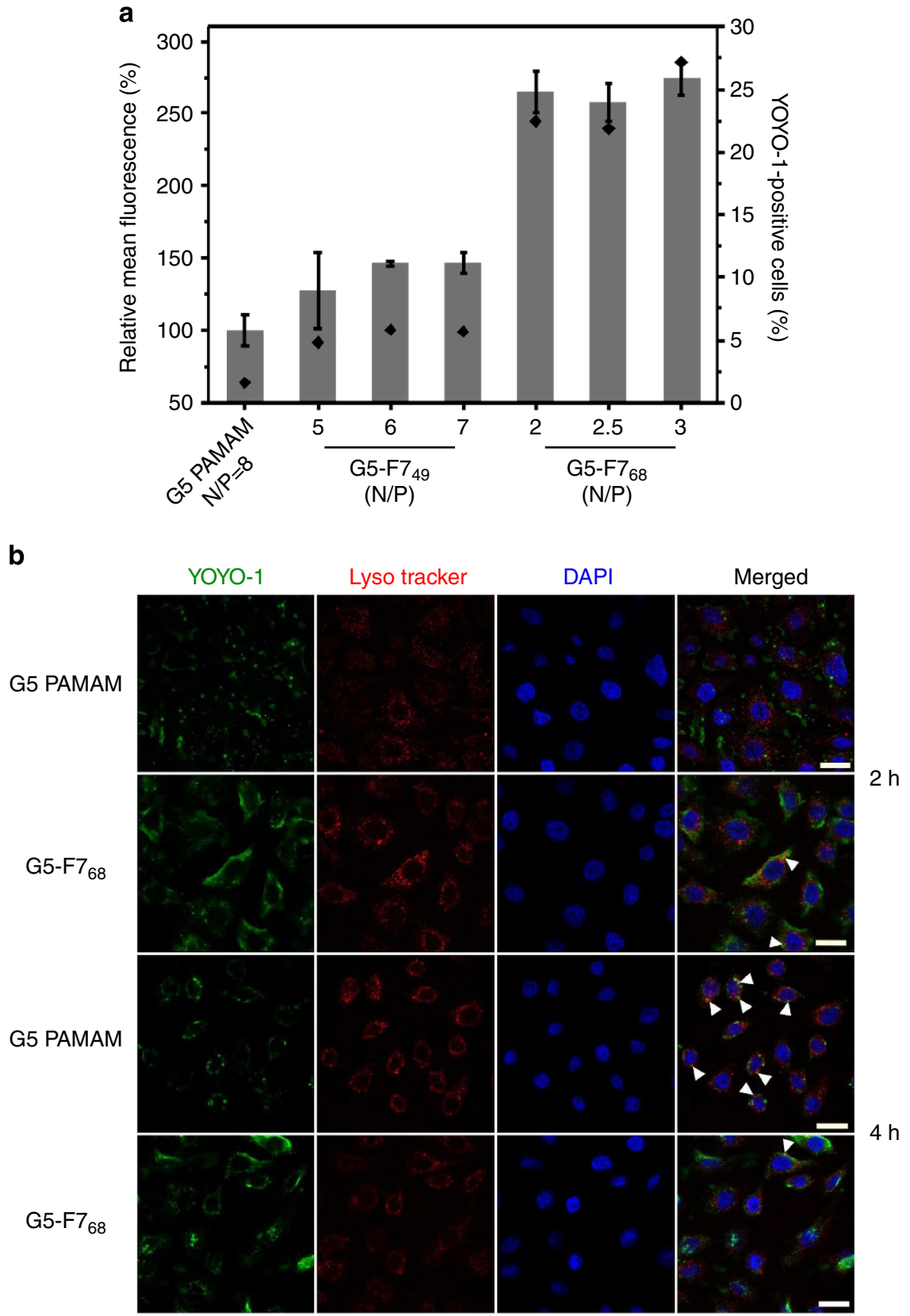

Figure 5 | Cellular uptake and endosomal escape abilities of fluorinated dendrimers in HeLa cells. (a) Cellular uptake of YOYO-1-labelled DNA delivered by $\mathrm{G} 5, \mathrm{G} 5-\mathrm{F}_{49}$ and $\mathrm{G} 5-\mathrm{F}_{68}$, respectively. The samples were incubated with HeLa cells for $1 \mathrm{~h}$. Error bars represent the s.e. ( $n=3$ ). Diamonds represent YOYO-1-positive cells (\%). (b) Confocal images of HeLa cells treated with G5-F768/DNA and G5/DNA polyplexes for 2 and 4 h, respectively. DNA was labelled with YOYO-1, the endosomes were stained with LysoTracker Red and the nuclei were stained with DAPI (4',6-diamidino-2-phenylindole). The arrows indicate co-localization of YOYO-1-labelled DNA and LysoTracker Red-stained endosomes in HeLa cells. Scale bar, $20 \mu \mathrm{m}$.

Gene transfection at a low N/P ratio ensures low cytotoxicity of the materials during gene transfection ${ }^{43}$. As shown in Fig. $4 \mathrm{a}$ and Supplementary Fig. 10, G5-F7 68 and G5-F7 49 show low cytotoxicity ( $>90 \%$ cell viability) on these cells. In addition, G5-F7 68 shows reproducible gene transfection in HeLa cells (Supplementary Fig. 11). These results suggest that G5-F7 68 is an efficient and biocompatible vector for the introduction of both EGFP and luciferase genes into a list of commonly used cells.

Transfection mechanisms of fluorinated dendrimers. To verify the role of fluorine on the high transfection efficacy of G5-F7 68 , we synthesized butyric acid-modified G5 dendrimer, termed
G5-H7 69 (Supplementary Fig. 12). As shown in Supplementary Fig. 13, G5-H7 69 shows extremely weak EGFP transfection efficacy on HEK293 and HeLa cells, suggesting that fluorine modified on dendrimer surface plays an essential role in the efficient gene transfection by $\mathrm{G} 5-\mathrm{F} 7_{68}$. We also tested the gene transfection efficacy of G5 dendrimer/perfluorobutyric acid physical mixture. As shown in Supplementary Fig. 14, the addition of perfluorobutyric acid does not enhance the gene transfection efficacy of G5 dendrimer, suggesting that perfluorobutyric acid should be covalently attached to dendrimer surface to yield an efficient gene vector, such as $\mathrm{G} 5-\mathrm{F}_{68}$.

To investigate the reason behind the high transfection efficacy of fluorinated dendrimers, we compared the cellular uptake and 
endosomal escape behaviours of G5/DNA and fluorinated G5/ DNA polyplexes. As shown in Fig. 5a, fluorination significantly improves the cellular uptake efficacy of DNA bound on G5 dendrimer. $\mathrm{G}^{-}-\mathrm{F}_{68}$ shows the highest efficacy on delivering DNA into HeLa cells. Fluorination also facilitates the endosomal escape of dendrimer/DNA polyplexes. The addition of bafilomycin Al, which inhibits the acidification in endosome and lysosome, can efficiently block the transfection of EGFP gene by G5-F7 68 , whereas the supplement of sucrose, a lysosomotropic agent that accelerates the escape of polyplexes from acidic vesicles, cannot further improve the transfection efficacy of G5F7 68 (Supplementary Fig. 15) ${ }^{44,45}$. The efficient cellular uptake and endosomal escape of $\mathrm{G} 5-\mathrm{F}_{68} / \mathrm{DNA}$ polyplexes are further confirmed by confocal microscopy. DNA (labelled with a green fluorescent dye, YOYO-1) delivered by G5-F7 68 showed efficient cellular uptake $(\sim 100 \%)$ in HeLa cells and most of the DNA escaped from the acidic vesicles stained by LysoTracker Red within $4 \mathrm{~h}$ (Fig. 5b); however, G5/DNA and G5-F7 ${ }_{49} / \mathrm{DNA}$ polyplexes shows less efficient cellular uptake than $\mathrm{G} 5-\mathrm{F}_{68} / \mathrm{DNA}$ polyplexes. Co-localizations of YOYO-1 within the acidic vesicles are observed for these polyplexes at $4 \mathrm{~h}$ (Fig. $5 \mathrm{~b}$ and Supplementary Fig. 16).
Fluorinated dendrimer shows excellent serum resistance. The gene transfection using fluorinated dendrimers in this study was conducted in medium containing $10 \%$ fetal bovine serum (FBS). As shown in Fig. 6, G5-F7 68 preserves efficient gene transfection (55.4\%) in HeLa cells even in medium containing $50 \% \mathrm{FBS}$, whereas Lipofectamine 2000 almost lost its transfection efficacy in medium containing $30 \%$ FBS. The excellent serum resistance of G5-F7 68 in gene delivery is attributed to the inactive surface of fluorinated dendrimer against serum proteins. This result shows encouraging gene delivery potential of $\mathrm{G}_{5}-\mathrm{F}_{68}$ for in vivo applications.

\section{Discussion}

The transfection efficacy of a polymeric gene vector depends on multiple parameters, that is, DNA condensation and protection, serum stability, cellular uptake efficiency, intracellular trafficking such as endosomal escape, DNA unpacking and nuclear entry $^{8}$. As demonstrated above, fluorination on dendrimers enhances cellular uptake, endosomal escape and serum resistance of the dendrimer/DNA polyplexes. It also modulates the DNA packing/unpacking ability of the dendrimers. As shown a
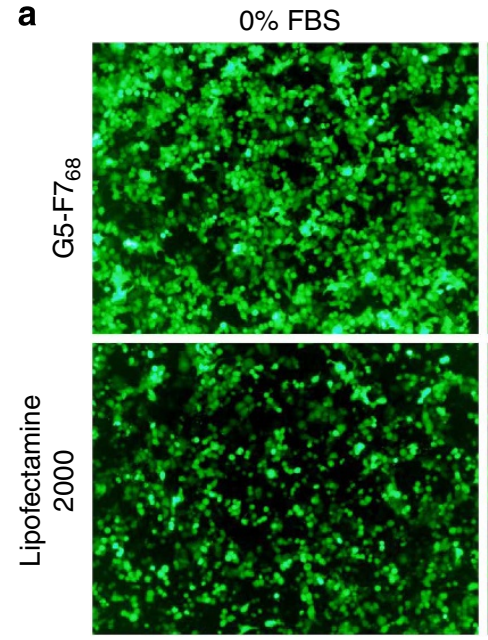

$10 \%$ FBS
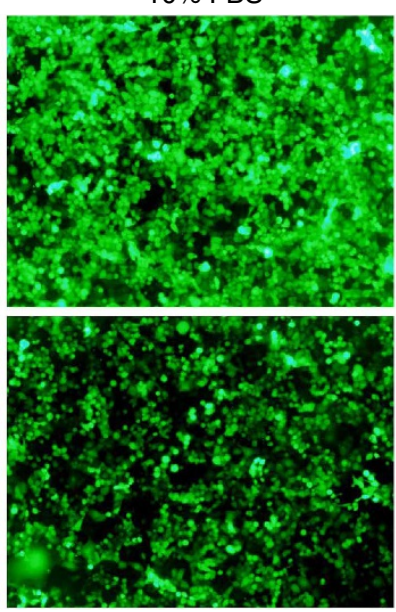

$30 \%$ FBS
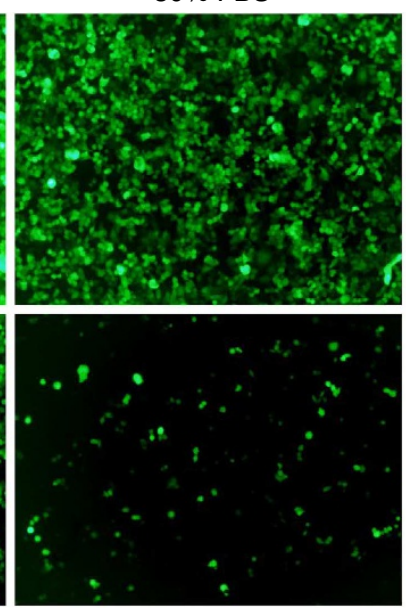

$50 \%$ FBS
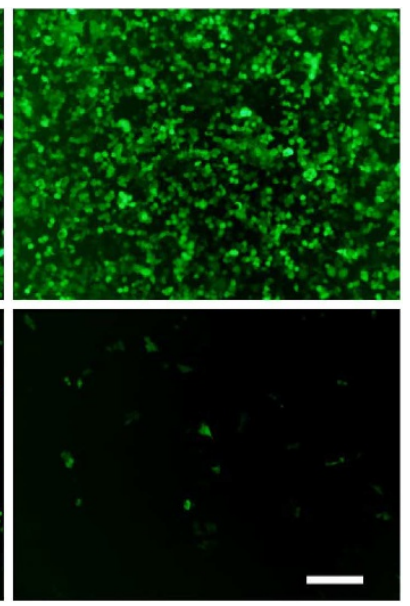

b

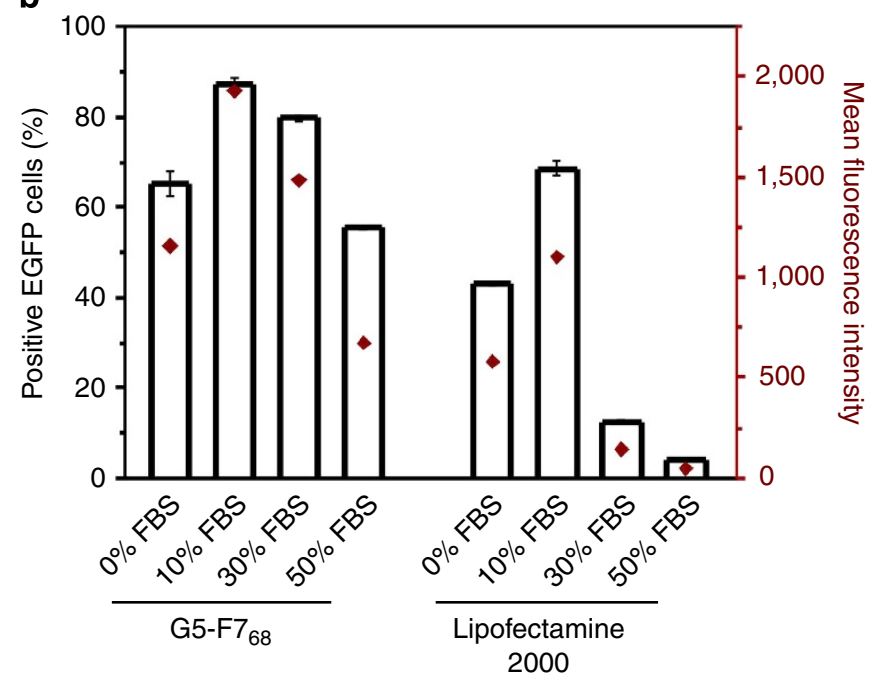

Figure 6 | Serum-resistance abilities of fluorinated dendrimers on EGFP gene transfection. EGFP expression in HeLa cells were performed using G5-F7 68 (N/P ratio at 2.5:1) and Lipofectamine 2000 in mediums containing 0\%, 10\%, 30\% and 50\% FBS, respectively (a). The EGFP gene transfection efficacy was determined by flow cytometry $(\mathbf{b})$. Error bars in $\mathbf{b}$ represent the s.e. $(n=3)$. Diamonds in $\mathbf{b}$ represent mean fluorescence intensity. Scale bar, $200 \mu \mathrm{m}$. 
in Supplementary Fig. 17, G5-F7 68 is easier to release its bound DNA in the presence of heparin than G5 dendrimer, owing to the low N/P ratio of G5-F7 68 /DNA polyplexes, to the reduced surface charge density on dendrimer surface after fluorination and to the inert surface of fluorinated dendrimer towards the bound DNA. If a plasmid DNA is loosely bound with the vector, it will be rapidly degraded by cytosolic nuclease. On the contrary, tight binding makes it difficult for DNA unpacking and leads to failed gene transfection $^{8}$. Dendrimer is such a material that has strong binding ability with DNA owing to the multivalency effect of the surface charges. The incorporation of an anionic polymer into the dendrimer/DNA polyplex can increase the DNA release rate and improve the transfection efficacy of cationic dendrimers ${ }^{46,47}$. Therefore, the easier DNA unpacking ability of G5-F7 68 compared with unmodified dendrimer might be one of the reasons to explain the dramatic efficacy of $\mathrm{G}_{5}-\mathrm{F}_{68}$ in gene delivery.

It is worth noting that perfluoroaliphatic acid is a protecting group for amines and the corresponding amide could be hydrolysed under basic conditions. Surprisingly, the stability of fluorinated dendrimer $\mathrm{G} 5-\mathrm{F}_{68}$ is excellent in aqueous solutions (Supplementary Fig. 18). G5-F7 68 maintains efficient gene transfection after the samples were stored at $-20^{\circ} \mathrm{C}$ for 8 months (Supplementary Fig. 11). Even if the fluorinated dendrimer is degraded in the cells, it will not induce additional toxicity on the transfected cells. As shown in Supplementary Fig. 19 , both G5-F7 68 and G5/heptafluorobutyric acid mixture (fully degraded products of $\mathrm{G} 5-\mathrm{F}_{68}$ ) are non-toxic on the cells, suggesting the excellent biocompatibility of the fluorinated dendrimer in gene delivery. One may ask why $\mathrm{G}_{5}-\mathrm{F}_{68}$ has a much higher transfection efficacy than other fluorinated dendrimers. A possible answer is suggested by a balance of several factors influencing the transfection efficacy, such as chain length of the perfluoroaliphatic acid, number of conjugated ligands and residual surface charges, and degradability of the fluorinated dendrimer. This phenomenon is also observed in the gene transfection efficacies of dendrimers modified with aliphatic acid of different chain lengths ${ }^{48}$. The exact reason behind this question needs further investigations on the fluorinated dendrimers.

In summary, fluorinated dendrimer $\mathrm{G} 5-\mathrm{F}_{68}$ achieves high gene transfection efficacy even at extremely low $\mathrm{N} / \mathrm{P}$ ratio around 0.5. The efficacy of G5-F7 68 is higher than several commercial gene reagents such as Lipofectamine 2000 and SuperFect in a list of cells. Low dose of the G5-F7 68 used ensures high viability of the cells during gene transfection. Fluorination removes the association of transfection efficacy and cytotoxicity for dendrimer-based gene delivery systems. The increased transfection efficacy of dendrimer after fluorination is mainly attributed to increased cellular uptake and facilitated endosomal escape of the polyplexes. In addition, $\mathrm{G}^{-}-\mathrm{F}_{68}$ shows excellent serum resistance and even exhibits high transfection efficacy in medium containing 50\% FBS. Not limited to G5 PAMAM dendrimer, fluorination also works well on other dendrimer generations (such as G4 PAMAM dendrimer, Supplementary Fig. 20), other dendrimer species (such as polypropylenimine dendrimer, Supplementary Fig. 21) and other cationic polymers (such as bPEI25K, Supplementary Fig. 22). All these fluorinated materials achieved high transfection efficacies at low N/P ratios. Taken together, G5- $7_{68}$ showed dramatic gene transfection efficacy in commonly used cell lines. Fluorination on polymers as well as other biomaterials can be used as a versatile tool in the development of efficient drug and gene carriers. Further work will be focused on investigating the exact mechanism of fluorination on gene delivery and evaluating the in vivo efficacy of fluorinated polymers such as G5-F7 68 on the delivery of therapeutic DNAs into tumour-bearing mice.

\section{Methods}

Materials. Ethylenediamine-cored G5 PAMAM dendrimer was purchased from Dendritech, Inc. (Midland, MI). Trifluoroacetic anhydride, pentafluoropropionic anhydride, heptafluorobutyric anhydride, nonafluorobutanesulphonic anhydride, butyric anhydride, bafilomycin Al and sucrose were purchased from Sigma-Aldrich (St Louis, MO). The chemicals were used as received without further purification.

Synthesis and characterization of fluorinated dendrimers. G5 PAMAM dendrimer and perfluoro acid anhydrides were dissolved in methanol at different molar ratios. The mixtures were stirred at room temperature for $48 \mathrm{~h}$ and dialysed against PBS buffer and distilled water. The products were lyophilized under vacuum to obtain fluorinated dendrimers as white gels. The number of remaining amine groups on each fluorinated G5 dendrimer was evaluated by a well-established ninhydrin assay (Supplementary Method). The numbers of perfluoro acid modified on G5 dendrimers are listed in Supplementary Table 1. The synthesis of butyric acid-modified G5 dendrimer was conducted using a similar method as described above, and the number of butyric acid modified on each G5 dendrimer was determined using ${ }^{1} \mathrm{H}$ NMR.

Characterization of fluorinated dendrimer/DNA polyplexes. Fluorinated dendrimers, including G5-F7 68 and G5-F7 49 , and unmodified G5 were mixed with DNA at different N/P ratios $(0: 1,0.5: 1,1: 1,2: 1,4: 1$ and 8:1) for $30 \mathrm{~min}$. The samples were electrophoresed on $1 \%$ agarose gels and stained by ethidium bromide for $1 \mathrm{~h}$. To compare the stability of G5-F7 ${ }_{68} / \mathrm{DNA}$ and G5/DNA, the polyplexes at an N/P ratio of $2: 1$ or $8: 1$ in the presence of different concentrations of heparin were also analysed using agarose gel electrophoresis. Size and zeta-potential of the fluorinated dendrimer/DNA polyplexes were further characterized at $25^{\circ} \mathrm{C}$ using dynamic light scattering (Zetasizer Nano ZS90, Malvern Zetasizer 3000).

Cell culture and gene transfection. HEK293 (a human embryonic kidney cell line, ATCC), HeLa (a human cervical carcinoma cell line, ATCC), NIH3T3 (mouse embryo fibroblasts, ATCC), COS-7 (a fibroblast-like cell line derived from monkey kidney, ATCC) and CHO (a Chinese hamster ovary cell line, ATCC) cells were cultured in DMEM (GIBCO) containing penicillin sulphate ( 100 units $\left.\mathrm{ml}^{-1}\right)$, streptomycin $\left(100 \mu \mathrm{g} \mathrm{ml}^{-1}\right)$ and $10 \%$ heat-inactived FBS (GIBCO). The cells were cultured in 24 -well plates for $24 \mathrm{~h}$ before in vitro gene transfection, followed by the incubation of dendrimer/DNA polyplexes $(0.8 \mu \mathrm{g}$ DNA, the polyplexes were prepared at different $\mathrm{N} / \mathrm{P}$ ratios and dissolved in $250 \mu \mathrm{l}$ DMEM containing $10 \% \mathrm{FBS}$ ) with the cells for $6 \mathrm{~h}$ Next, $500 \mu \mathrm{l}$ fresh DMEM containing 10\% FBS were added. Commercial transfection reagents such as Lipofectamine 2000 and SuperFect were used as controls, and gene transfection by these materials were conducted according to the product's protocols (Invitrogen). The optimized N/P ratios for other controls such as unmodified G5 PAMAM dendrimer, bPEI25K, G5-Lys 130 and G5-Arg ${ }_{108}$ were screened before their comparisons on transfection efficacy with fluorinated dendrimers.

The gene transfection experiments were conducted for $24 \mathrm{~h}$ (HEK293 cells) or $48 \mathrm{~h}$ (other cell lines). The expression of EGFP plasmid in the cells was directly observed by a fluorescent microscopy (Olympus, Japan), and the transfection efficacy was quantitatively measured using flow cytometry. The determination of luciferase plasmid expression was performed according to the manufacturer's protocols (Promega). The luciferase activities were normalized with respect to protein concentrations (relative luciferase light units per milligram protein).

To investigate the serum resistance of the fluorinated dendrimers, gene transfection experiments for G5-F7 68 and Lipofectamine 2000 were also conducted on HeLa cells in medium containing 0, 10, 30 and 50\% FBS.

Cellular uptake and gene transfection mechanisms. To investigate the cellular uptake efficacies of fluorinated dendrimer/DNA and unmodified dendrimer/DNA polyplexes, DNA was labelled with YOYO-1 (Y3601, Invitrogen) for $10 \mathrm{~min}$ before the preparation of polyplexes; HeLa cells were then incubated with the YOYO-1labelled polyplexes for $1 \mathrm{~h}$ and uptake efficacy was analysed using flow cytometry.

To investigate the effect of fluorination on the endosomal escape of dendrimer/ DNA polyplexes, YOYO-1 was used to monitor intracellular trafficking behaviours of the polyplexes. The cells were incubated with the polyplexes for 2 or $4 \mathrm{~h}$. The acidic vesicles and the nuclei of HeLa cells were then stained with a red fluorescent dye (LysoTracker Red, DND-99, Invitrogen) and a blue fluorescent dye $\left(4^{\prime}, 6-\right.$ diamidino-2-phenylindole), respectively. Co-localizations of the polyplexes with acidic vesicles in HeLa cells were observed by a laser scanning confocal microscopy (Olympus FV1000). Sucrose and bafilomycin A1 was also used to evaluate the endosome escape abilities of the polyplexes. In general, sucrose $(5 \mathrm{mM})$ and bafilomycin A1 $(300 \mathrm{nM})$ were applied to the cells $1 \mathrm{~h}$ before the addition of dendrimer/EGFP plasmid polyplexes. The transfection experiments were conducted as described above. EGFP expressions in HeLa cells treated with and without sucrose or bafilomycin Al were examined by flow cytometry. The polyplexes for unmodified G5 PAMAM dendrimer and fluorinated dendrimers in the cellular uptake and endosomal escape experiments are prepared at their optimal N/P ratios on HeLa Cells.

Cytotoxicity analysis. To determine the cytotoxicity of $\mathrm{G} 5-\mathrm{F} 7_{49}$ and $\mathrm{G} 5-\mathrm{F} 7_{68}$, an MTT (3-(4,5-dimethylthiazol-2-yl)-2,5-diphenyltetrazolium bromide) assay was 
performed in 96-well plates according to well-established protocols. The cells were incubated with fluorinated dendrimers at concentrations equal to that used in gene transfection experiments for $24 \mathrm{~h}$ or $48 \mathrm{~h}$. Lipofectamine 2000 and unmodified G5 dendrimer were used as controls. Five repeats were conducted for each sample and the data were given as mean \pm s.e.m. and analysed by Students' $t$-test.

\section{References}

1. Ornelas-Megiatto, C., Wich, P. R. \& Frechet, J. M. Polyphosphonium polymers for siRNA delivery: an efficient and nontoxic alternative to polyammonium carriers. J. Am. Chem. Soc. 134, 1902-1905 (2012).

2. Cui, L. N. et al. Conjugation chemistry through acetals toward a dextran-based delivery system for controlled release of siRNA. J. Am. Chem. Soc. 134, 15840-15848 (2012).

3. Cohen, J. L. et al. Acid-degradable cationic dextran particles for the delivery of siRNA therapeutics. Bioconjugate Chem. 22, 1056-1065 (2011).

4. Cohen, J. A. et al. Acetal-modified dextran microparticles with controlled degradation kinetics and surface functionality for gene delivery in phagocytic and non-phagocytic cells. Adv. Mater. 22, 3593-3597 (2010).

5. Mastrobattista, E. \& Hennink, W. E. Polymers for gene delivery: charged for success. Nat. Mater. 11, 10-12 (2011).

6. Anderson, D. G. et al. A polymer library approach to suicide gene therapy for cancer. Proc. Natl Acad. Sci. 101, 16028-16033 (2004).

7. Siegwart, D. J. et al. Combinatorial synthesis of chemically diverse core-shell nanoparticles for intracellular delivery. Proc. Natl Acad. Sci. 108, 12996-13001 (2011).

8. Wagner, E. Strategies to improve DNA polyplexes for in vivo gene transfer: will 'artificial viruses' be the answer? Pharm. Res. 21, 8-14 (2004).

9. Liu, H., Wang, H., Yang, W. \& Cheng, Y. Disulfide cross-linked low generation dendrimers with high gene transfection efficacy, low cytotoxicity, and low cost. J. Am. Chem. Soc. 134, 17680-17687 (2012).

10. Zhou, J. et al. Biodegradable poly(amine-co-ester) terpolymers for targeted gene delivery. Nat. Mater. 11, 82-90 (2011).

11. Woodrow, K. A. et al. Intravaginal gene silencing using biodegradable polymer nanoparticles densely loaded with small-interfering RNA. Nat. Mater. 8, 526-533 (2009).

12. Zeng, H., Little, H. C., Tiambeng, T. N., Williams, G. A. \& Guan, Z Multifunctional dendronized peptide polymer platform for safe and effective siRNA delivery. J. Am. Chem. Soc. 135, 4962-4965 (2013).

13. Yuba, E. et al. Effect of unsaturated alkyl chains on transfection activity of poly (amidoamine) dendron-bearing lipids. J. Control. Release 160, 552-560 (2012).

14. Kulkarni, A., DeFrees, K., Hyun, S. H. \& Thompson, D. H. Pendant polymer:amino-beta-cyclodextrin:siRNA guest:host nanoparticles as efficient vectors for gene silencing. J. Am. Chem. Soc. 134, 7596-7599 (2012).

15. Huang, S. et al. Dual targeting effect of Angiopep-2-modified, DNA-loaded nanoparticles for glioma. Biomaterials 32, 6832-6838 (2011).

16. Patil, M. L., Zhang, M. \& Minko, T. Multifunctional triblock Nanocarrier (PAMAM-PEG-PLL) for the efficient intracellular siRNA delivery and gene silencing. ACS Nano 5, 1877-1887 (2011).

17. Su, H. et al. Amphiphilic starlike dextran wrapped superparamagnetic iron oxide nanoparticle clsuters as effective magnetic resonance imaging probes. Biomaterials 34, 1193-1203 (2013).

18. Muller, K., Faeh, C. \& Diederich, F. Fluorine in pharmaceuticals: looking beyond intuition. Science 317, 1881-1886 (2007).

19. Purser, S., Moore, P. R., Swallow, S. \& Gouverneur, V. Fluorine in medicinal chemistry. Chem. Soc. Rev. 37, 320-330 (2008).

20. Liu, W. et al. Oxidative aliphatic C-H fluorination with fluoride ion catalyzed by a manganese porphyrin. Science 337, 1322-1325 (2012).

21. Buer, B. C., Meagher, J. L., Stuckey, J. A. \& Marsh, E. N. Structural basis for the enhanced stability of highly fluorinated proteins. Proc. Natl Acad. Sci. 109, 4810-4815 (2012).

22. Wang, Y. et al. Fluorinated graphene for promoting neuro-induction of stem cells. Adv. Mater. 24, 4285-4290 (2012).

23. Takaoka, Y. et al. Self-assembling nanoprobes that display off/on $19 \mathrm{~F}$ nuclear magnetic resonance signals for protein detection and imaging. Nat. Chem. 1, 557-561 (2009).

24. Lee, E. et al. A fluoride-derived electrophilic late-stage fluorination reagent for PET imaging. Science 334, 639-642 (2011).

25. Horvath, I. T. \& Rabai, J. Facile catalyst separation without water: fluorous biphase hydroformylation of olefins. Science 266, 72-75 (1994).

26. Xiong, S. D. et al. Cationic fluorine-containing amphiphilic graft copolymers as DNA carriers. Biomaterials 31, 2673-2685 (2010).

27. Kasuya, M. C. Z., Nakano, S., Katayama, R. \& Hatanaka, K. Evaluation of the hydrophobicity of perfluoroalkyl chains in amphiphilic compounds that are incorporated into cell membrane. J. Fluorine Chem. 132, 202-206 (2011).

28. Neil, E. \& Marsh, G. Towards the nonstick egg: designing fluorous proteins. Chem. Biol. 7, R153-R157 (2000).

29. Percec, V., Imam, M. R., Peterca, M. \& Leowanawat, P. Self-organizable vesicular columns assembled from polymers dendronized with semifluorinated
Janus dendrimers act as reverse thermal actuators. J. Am. Chem. Soc. 134, 4408-4420 (2012).

30. Percec, V. et al. Self-organization of supramolecular helical dendrimers into complex electronic materials. Nature 419, 384-387 (2002).

31. Brittain, S. M., Ficarro, S. B., Brock, A. \& Peters, E. C. Enrichment and analysis of peptide subsets using fluorous affinity tags and mass spectrometry. Nat. Biotechnol. 23, 463-468 (2005).

32. Tomalia, D. A. Interview: An architectural journey: from trees, dendrons/ dendrimers to nanomedicine. Interview by Hannah Stanwix. Nanomedicine 7, 953-956 (2012).

33. Tomalia, D. A. Dendritic effects: dependency of dendritic nano-periodic property patterns on critical nanoscale design parameters (CNDPs). N. J. Chem 36, 264-281 (2012)

34. Menjoge, A. R., Kannan, R. M. \& Tomalia, D. A. Dendrimer-based drug and imaging conjugates: design considerations for nanomedical applications. Drug Discov. Today 15, 171-185 (2010).

35. Almutairi, A. et al. Biodegradable dendritic positron-emitting nanoprobes for the noninvasive imaging of angiogenesis. Proc. Natl Acad. Sci. 106, 685-690 (2009).

36. Lee, C. C., MacKay, J. A., Frechet, J. M. \& Szoka, F. C. Designing dendrimers for biological applications. Nat. Biotechnol. 23, 1517-1526 (2005).

37. Lee, C. C. et al. A single dose of doxorubicin-functionalized bow-tie dendrimer cures mice bearing C-26 colon carcinomas. Proc. Natl Acad. Sci. 103, 16649-16654 (2006).

38. Caminade, A. M., Turrin, C. O., Sutra, P. \& Majoral, J. P. Fluorinated dendrimers. Curr. Opin. Colloid. Interf. Sci. 8, 282-295 (2003).

39. Criscione, J. M. et al. Self-assembly of $\mathrm{pH}$-responsive fluorinated dendrimerbased particulates for drug delivery and noninvasive imaging. Biomaterials $\mathbf{3 0}$, 3946-3955 (2009).

40. Vierling, P., Santaella, C. \& Greiner, J. Highly fluorinated amphiphiles as drug and gene carrier and delivery systems. J. Fluorine Chem. 107, 337-354 (2001).

41. Klein, E. et al. "HFP" fluorinated cationic lipids for enhanced lipoplex stability and gene delivery. Bioconjugate Chem. 21, 360-371 (2010).

42. Kim, T. I., Bai, C. Z., Nam, K. \& Park, J. S. Comparison between arginine conjugated PAMAM dendrimers with structural diversity for gene delivery systems. J. Control. Release 136, 132-139 (2009).

43. Alameh, M. et al. Low molecular weight chitosan nanoparticulate system at low $\mathrm{N}: \mathrm{P}$ ratio for nontoxic polynucleotide delivery. Int. J. Nanomed. 7, 1399-1414 (2012).

44. Breunig, M., Lungwitz, U., Liebl, R. \& Goepferich, A. Breaking up the correlation between efficacy and toxicity for nonviral gene delivery. Proc. Natl Acad. Sci. 104, 14454-14459 (2007).

45. Wen, Y. et al. Serum tolerance and endosomal escape capacity of histidinemodified pDNA-loaded complexes based on polyamidoamine dendrimer derivatives. Biomaterials 33, 8111-8121 (2012).

46. Zhang, Q., Chen, S., Zhuo, R. X., Zhang, X. Z. \& Cheng, S. X. Self-assembled terplexes for targeted gene delivery with improved transfection. Bioconjugate Chem. 21, 2086-2092 (2010).

47. Maksimenko, A. V. et al. Optimisation of dendrimer-mediated gene transfer by anionic oligomers. J. Gene Med. 5, 61-71 (2003).

48. Santos, J. L. et al. Functionalization of poly(amidoamine) dendrimers with hydrophobic chains for improved gene delivery in mesenchymal stem cells. J. Control. Release 144, 55-64 (2010).

\section{Acknowledgements}

This work was supported by the Science and Technology of Shanghai Municipality (11DZ2260300), the National Natural Science Foundation of China (numbers 21274044 and 21322405) and the Shanghai Rising-star Program (13QA1401500).

\section{Author contributions}

M.W. synthesized the materials, primarily screened their transfection efficacies and wrote the manuscript. H.L. performed most of the gene transfection and cytotoxicity experiments, and analysed the data. L.L. performed part of the experiments on gene transfection mechanism. Y.C. designed and supervised the study, and wrote the manuscript

\section{Additional information}

Supplementary Information accompanies this paper at http://www.nature.com/ naturecommunications

Competing financial interests: The authors declare no competing financial interests.

Reprints and permission information is available online at http://npg.nature.com/ reprintsandpermissions/

How to cite this article: Wang, M. et al. A fluorinated dendrimer achieves excellent gen transfection efficacy at extremely low nitrogen to phosphorus ratios. Nat. Commun. 5:3053 doi: 10.1038/ncomms4053 (2014). 